\title{
Design, Detection and Management of Mechanical Security Code Manhole Cover
}

\author{
Zhonghua $\mathrm{Su}^{1}$, Xiang $\mathrm{Yu}^{1}$, Zhangcheng Yang ${ }^{1}$, Heng Kang ${ }^{2}$ and Jian $\mathrm{Ou}^{3}$ \\ ${ }^{1}$ Chongqing Institute of Metrology and Quality Inspection, Chongqing, 401123 \\ ${ }^{2}$ Chongqing Municipal Facilities Administration, Chongqing, 400015 \\ ${ }^{3}$ Chongqing Zhongtian Electromechanical Technology Co., Ltd, Chongqing, 400039
}

\begin{abstract}
In this paper, the researches and the application of manhole over as well as the existing problems have been described. The mechanical security code manhole cover with the built-in locking mechanism has been designed. The locking mechanism can withstand the muddy water immersion test, the password design test, the low temperature alternating cycle test, the failure load test and the vibration test. The manhole cover is equipped with an electric key and a mechanical key. The electric key automatically opens the code lock by receiving the password. The mechanical key demands to manually adjust the dial to open the code lock. The key and the password are respectively managed by different people.
\end{abstract}

Keywords: Password manhole cover; Locking mechanism; Electric key; Mechanical key.

\section{Introduction (research and application of manhole cover and intelligent manhole cover)}

The manhole cover is the entrance and exit enclosure to the underground facilities. It is necessary to install the manhole cover in the places where the tap water, the cable TV, the drainage, the telecommunications, the China Mobile, the Unicom, the electricity, the gas, the heat, the fire protection, the sanitation and other public facilities are installed ${ }^{[1]}$. At present, the manhole covers are mostly the traditional low-end products at home and abroad. The common problems are that the covers are easily stolen and lost to form the road traps to cause casualties, the noise is disturbing people and the digital intelligent management is unable to be realized. Especially the lack of crime prevention function, there is a public safety hazard ${ }^{[2-4]}$.

With the development of Internet and related fields, the development trend of manhole cover should have the function of digital intelligent management in addition to the basic functions such as the simple structure, the reliable operation, the strong environmental adaptability, the anti-settlement and the anti-vibration. In particular, the manhole cover and the safety of system have become the important issues that the industry and the relevant government departments have actively considered ${ }^{[5-6]}$.

In recent years, the intelligent manhole covers of the Internet of things have been developed at home and abroad, which has overcome some shortcomings of the traditional manhole covers in theory, realized the remote control and alarming, easily managed and maintained and enhanced the security, etc. However, the cost of production and management of this kind of manhole cover is especially high. The electronic components in the manhole cover cannot guarantee the normal operation in extreme harsh environment such as cold and hot weather, sunshine and rain, heavy load impact, etc. It is easy to cause the false alarm or it is not opened by itself, causing great confusion to the users. Even if the normal alarm is also an accident post-processing. If there are consequences, it has been formed. The remote control system is easy to become the target of hacker attack. Instead, there will be greater safety risks ${ }^{[7-8]}$.

In view of the above problems, a set of mechanical security code manhole cover with the characteristics of low cost, strong weathering resistance, anti-steal, anti-settlement, anti-vibration and digital management has been designed in this paper.

\section{Design of mechanical security code manhole cover}

Compared with the manhole cover in GB/T 23858-2009 standard, the mechanical security code manhole cover designed in this paper mainly increases the password locking mechanism and the opening key. The detailed contents are as follows. 


\subsection{Password locking mechanism}

The design of the password locking mechanism is shown in Figure 1, in which the lock core is installed on the sinking hole of the manhole cover and the lock core can be rotated 360 degrees. The cipher plate is fixedly connected to the lock core. A pin hole is arranged at different angles on the surface of the cipher plate. The dowel pin is inserted into one of the pin holes. The dowel pin and the cipher disk rotate with the rotation of the lock core. In the process of rotation, the dowel pin will be not rotated due to the contact of the dowel pin block embedded in the manhole cover above the cipher disk. This is the 0 degree of the cipher disk. Rotating the cipher disk in the opposite direction, when the gap and the lock tongue in the cipher disk are in the alignment, the manhole cover can be opened. This is the opening angle of the cipher disk. Before the manhole cover is installed, the opening angle of the cipher disk can be adjusted through adjusting the position of the dowel pin. The parameters of the opening angle are usually retained in the manhole cover management system.

The mechanical security code manhole cover adopts the pure mechanical password mechanism, which can adapt to all kinds of the bad environment. Each manhole cover has two sets of cipher institutions at least and the number of the cipher groups is more than 800 groups. The illegal opening time is more than one hour. The security is strong, the reliability is high and the stability is good.

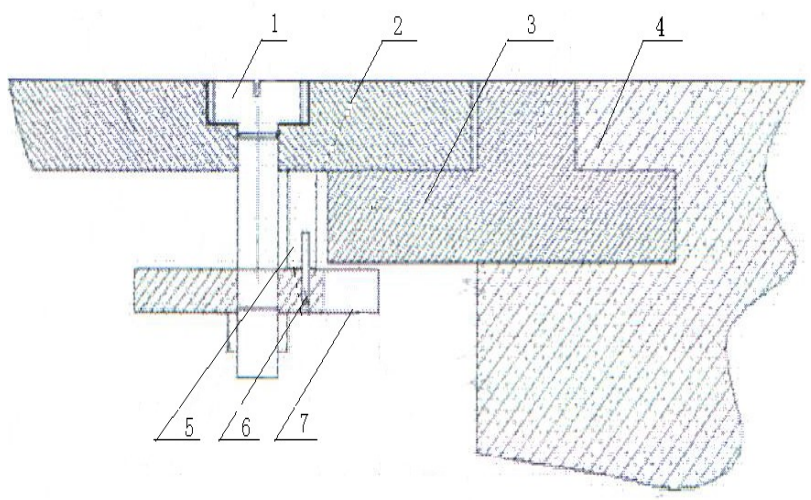

Figure 1. Password locking mechanism.

(1-lock core, 2-manhole cover, 3- lock tongue, 4-manhole seat, 5-dowel pin block, 6-dowel pin, 7-cipher disk.)

\subsection{Opening key}

For safety emergency reasons, two types of the manhole opening keys are designed in the design process, namely, the electric key and the mechanical key. When the management system fails and the electric key cannot be used, the emergency mechanical key can be used.

In Figure 2, the electric key is a combination of the fingerprint recognizer, the signal receiving controller, the motor and the opening end. The user needs to carry out the fingerprint identification before using the electric key. When the identification is successful, the opening end is inserted into the lock core. It is required to open the signal receiving controller and receive the opening password sent by the management system. The signal receiving controller can automatically control the angle of the motor rotation according to the received password, thus realizing the opening of the cipher lock.

In Figure 3, the mechanical key is combined with the dial disk and the opening end. The dial disk can be adjusted to the corresponding scale and positioned according to the backup password. Afterwards, the opening end is inserted into the lock core and rotated to the positioning scale to realize the opening of the cipher lock.

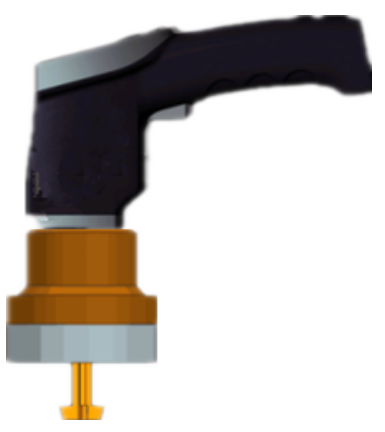

Figure 2. Electric key.

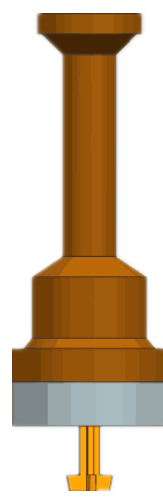

Figure 3. Mechanical key.

\section{Parameter detection of the password locking mechanism}

Compared with the manhole cover in GB/T 23858-2009, the mechanical security password manhole cover designed in this paper mainly increases the password locking mechanism. Therefore, the parameter index and the detection method of the password locking mechanism will be elaborated in detail.

\subsection{Muddy water immersion test}

Under the normal temperature, a set of codes are randomly selected. When the manhole cover is locked, the whole manhole cover is soaked in the muddy water (the proportion of the muddy and the water is 1:10). The muddy water should rise above the top of the manhole cover for $50 \mathrm{~mm}$ at least and it demands to be stirred for one time every 24 hours. After immersion for 168 hours, the manhole cover is opened with special key to check whether the locking mechanism is flexible and whether the manhole cover can be smoothly opened. If the manhole cover can be successfully opened, it will be qualified.

\subsection{Password design test}

In view of the number of cipher mechanisms, the number of the cryptographic mechanisms is not less than 2 sets. The total number of the cipher mechanisms on each manhole cover is not less than 800 groups. With the different combination of passwords, it will be qualified when the opening time of the manhole cover exceeds one hour. 


\subsection{High and low temperature resistance alternating cycle tests and destructive loading test}

The cipher mechanism is kept in $(-40 \pm 5){ }^{\circ} \mathrm{C}$ for 5 hours, then risen to $(85 \pm 5){ }^{\circ} \mathrm{C}$ in $30 \mathrm{~min}$ for 5 hours, and then cooled to room temperature. This is one cycle. After five cycles, it is necessary to check whether the cipher mechanism is flexible and whether there is crack and deformation. The destructive loading test of $5 \mathrm{kN}$ is carried out on the password locking mechanism after passing the test. After the test, if the password locking mechanism is not damaged, it will be qualified.

\subsection{Vibration test}

In the locking state, the locking mechanism records the relative positions of the lock tongue and the manhole cover and respectively marks the lines. The vibration test is carried out at the amplitude of $(0.5 \pm 0.05) \mathrm{mm}$ and the frequency of $(50 \pm 5) \mathrm{Hz}$. The cumulative time is not less than 12 hours. It is required to check the mark lines of the lock tongue and the manhole cover. When the relative displacement angle between the mark lines is less than 15 degrees, the locking mechanism will be qualified.

\section{Manhole cover management}

\subsection{Manhole cover management system}

The manhole cover management system includes the following functions: the login account, the login password, the input of the manhole cover information, the search of the manhole cover information, the modification and the deletion of the manhole cover information and the navigation of the manhole cover position. The manhole cover information mainly includes the manhole cover code, the specifications and models, the installation site photos, the installation address, the location information, the ownership unit, the responsible personnel and contact number, the in-well facilities, the installation time, the overhaul time and the setting and the sending of password (with permission settings), etc. The manhole cover information is inputted into the manhole cover management system when the manhole cover is installed by the management unit ${ }^{[9-10]}$.

\subsection{Opening of electric key}

When the electric key opens the manhole cover, the backstage of the management system provides the password that is transmitted to the electric key through the hand-held terminal wireless. Then, the master personnel of the electric key opens the lock. The person who has the electric key does not know the opening password. The management system backstage personnel know the password but cannot master the key.

\subsection{Opening of the emergency mechanical key}

When the management system fails and the electric key cannot be used, the emergency mechanical key can be used and the manhole cover can be opened by the password provided by the management system backup.

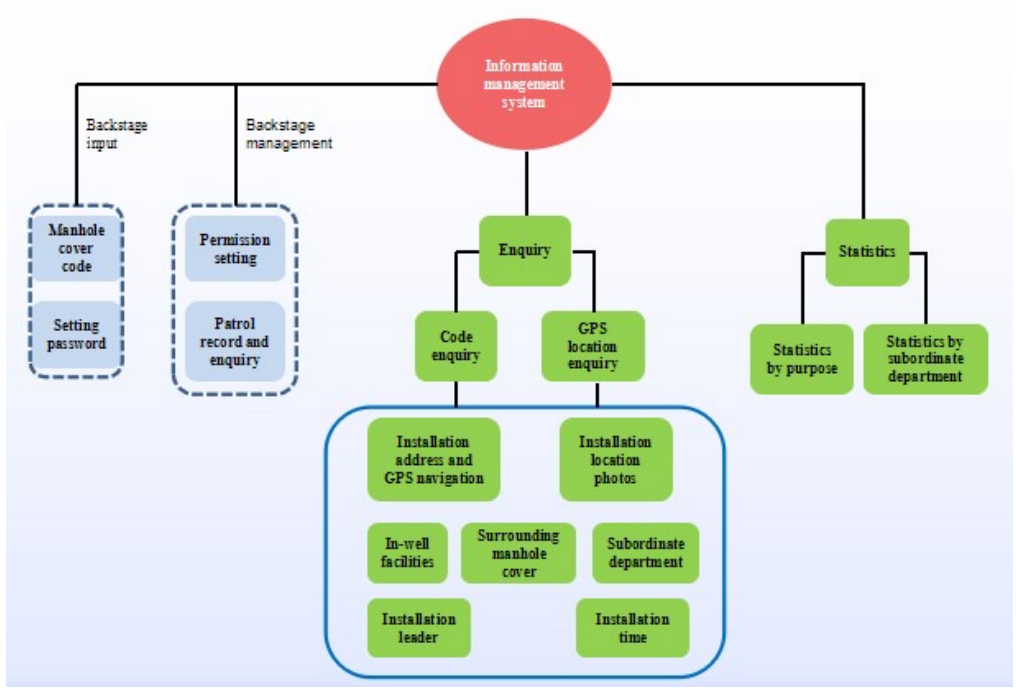

Figure 4. Manhole cover management system.

\section{Conclusion}

In view of the common problems of the existing manhole cover products, a set of mechanical security code manhole cover with the characteristics of low cost, strong weathering resistance, anti-steal, anti-settlement, anti-vibration and digital management has been designed in this paper. The key difference between this manhole cover and the conventional manhole cover is that it is equipped with the code locking mechanism under the manhole cover. This locking mechanism can withstand the muddy water immersion test, the password design test, the high and low temperature resistance alternating cycle test, the destructive loading test and the vibration test. 
The mechanical security password manhole cover is equipped with an electric key and a mechanical key. The electric key automatically opens the code lock by receiving the password. The mechanical emergency key needs to manually adjust the dial disk to open the code lock. The keys and the passwords are managed by different people.

\section{References}

1. Dai Sunfang. Research on Real Time Monitoring and Information Management Technology of Road Manhole Cover [J]. China Municipal Engineering, 2016 (6): 104-105.

2. Ren Anhu, Bao Honghai. Design of Safety Monitoring System of City Road Manhole Cover Based on Zigbee [J]. Intelligent Processing and Application, 2014 (11): 81.

3. Xu Wenqing, Xie Zhaoyan, Men Xing, Wang Jixiang. Anti-theft monitoring technology of manhole cover based on ZigBee and non-contact power supply and its application $[\mathrm{J}]$. Journal of Safety Science and Technology, 2012 (11): 173-174.
4. Liu Jianping, Wang Zhihua, Zhao Wei. Status and recommendations of the inspection well covers $[\mathrm{J}]$. Heilongjiang Science and Technology of Water Conservancyl, 2002, (3) :34.

5. Huang Lin. Research on the Real Time Inspection Technique of Scenting Well Based on WSN [J]. Hangzhou Dianzi University, 2013: 1-5.

6. Li Yasheng. Design of Manhole Cover Monitoring System Based on Infrared Detection [J]. Digital Technology and Application, 2015: 174-175.

7. Dang Lei, Pan Liangbo, Tao Weixiang. Research and Application of Monitoring and Warning Platform of City Manhole Cover [J]. Office Automation Journal, 2014 (10): 356-358.

8. Peng Bin, $\mathrm{Xu}$ Yingcheng, Ning Xiuli. Risk Analysis of Quality and Safety of Cover Products [J]. Standard Science, 2016 (6): 62-66.

9. Zheng Fengshou, Zhou Wen, Song Yongming. Intelligent Management of City Manhole Cover[J]. Surveying and Mapping Bulletin, 2013: 55-58.

10. Li Xianghong. Discussion on the Management Problems about Manholde Cover on Urban Road [J]. Municipal Engineering Technology, 2009 (11): 560-563. 